\title{
Visual Hallucinations Associated with Dorsal Thalamic Stroke
}

\section{JORGE A. BRENES-SALAZAR ${ }^{1}$}

A 51 year old male with a history of Type 2 diabetes mellitus and hypertension was admitted to the Medicine Service complaining of realistic visual hallucinations for a week, consisting of "unfamiliar dead people" and "dogs". On physical examination, he had no evidence of psychomotor agitation, his affect, judgment and thought processes were appropriate. No focal neurologic deficits were noted. Infectious and toxicology workup was unrevealing. MRI of the brainin axial, sagittal and coronal views identified the presence of a hypodense circular area in the dorsal right thalamus (Figure 1); furthermore, T2 sequences showed a hyperintense signal (Figure $2 \mathrm{~A}$ ), with concordant hypointensity in diffusion-weighted
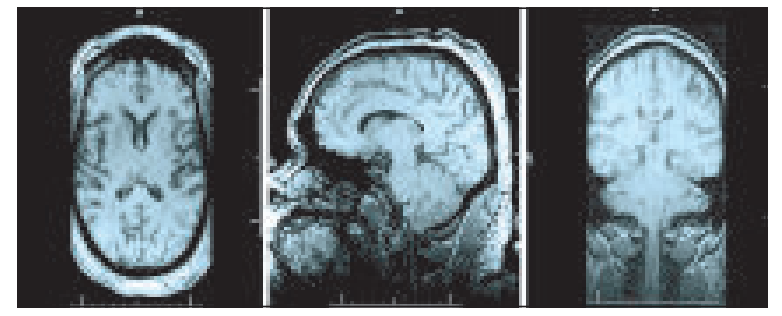

images (Figure 2 B), consistent with a subacute evolving stroke.

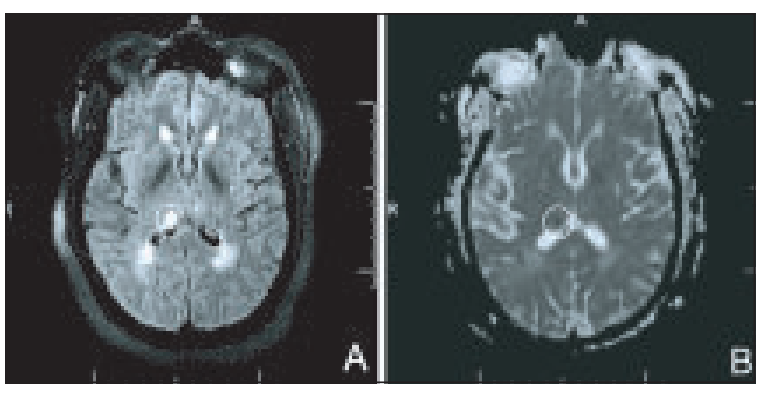

Vascular syndromes of the thalamus can have a variety of sensorimotor and behavioral presentations depending on the affected nuclei ${ }^{1}$; tuberothalamic infarcts can lead to visual processing deficitsincluding hallucinations, in the absence of motor deficits.

\section{References:}

1. Schmahmann, JD. Vascular syndromes of the thalamus. Stroke 2003; $34: 2264-2278$

1. MD, from the Department of Medicine, Division of Cardiovascular Diseases, Mayo Clinic Rochester, MN Correspondence: Jorge A Brenes-Salazar, MD. Mayo Clinic. 200 First St. SW, Rochester, MN 55905. Phone: 507-261-4644. Fax: 888664-1253. Email: brenessalazar.jorge@mayo.edu 